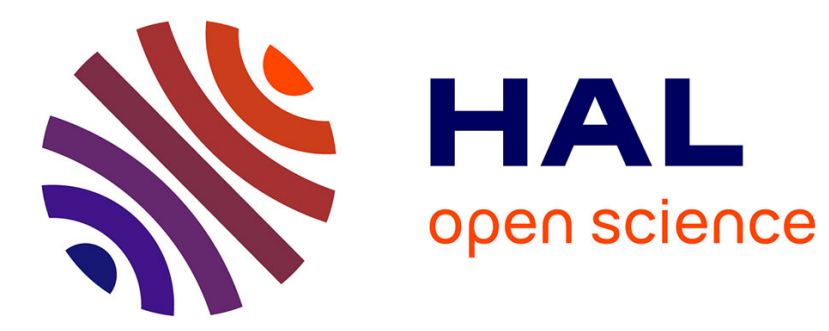

\title{
3D Coronary vessel tracking in x-ray projections
}

Emmanuelle Poulain, Grégoire Malandain, Régis Vaillant

\section{To cite this version:}

Emmanuelle Poulain, Grégoire Malandain, Régis Vaillant. 3D Coronary vessel tracking in x-ray projections. International Conference on Functional Imaging and Modeling of the Heart (FIMH), Jun 2017, Toronto, Canada. pp.204-215, 10.1007/978-3-319-59448-4_20 . hal-01502128

\section{HAL Id: hal-01502128 \\ https://hal.inria.fr/hal-01502128}

Submitted on 5 Apr 2017

HAL is a multi-disciplinary open access archive for the deposit and dissemination of scientific research documents, whether they are published or not. The documents may come from teaching and research institutions in France or abroad, or from public or private research centers.
L'archive ouverte pluridisciplinaire HAL, est destinée au dépôt et à la diffusion de documents scientifiques de niveau recherche, publiés ou non, émanant des établissements d'enseignement et de recherche français ou étrangers, des laboratoires publics ou privés. 


\title{
3D Coronary vessel tracking in x-ray projections
}

\author{
Emmanuelle Poulain ${ }^{1,2}$, Grégoire Malandain ${ }^{2}$, and Régis Vaillant ${ }^{1}$ \\ 1 GE Healthcare, 78530 Buc, France \\ 2 Université Côte d'Azur, Inria, CNRS, I3S, France \\ \{emmanuelle.poulain, regis.vaillant\}@ge.com \\ gregoire.malandain@inria.fr
}

\begin{abstract}
Fusing pre-operative CT angiography with per-operative angiographic and fluoroscopic images is considered by physicians as a potentially useful tool for improved guidance. To be adopted, this tool requires the development of tracking methods adapted to the deformations of the arteries caused by the cardiac motion. Here, we propose a 3D/2D temporal tracking of one coronary vessel, based on a spline deformation, using pairings with a controlled $2 \mathrm{D}$ stretching or contraction along the paired curves and a preservation of the length of the 3D curve. Experiments were conducted on a database of 10 vessels from 5 distinct patients, with dedicated metrics assessing both the global registration and the local coherency of the position along the vessel. The proposed results demonstrate the efficiency of the proposed method, with an average standard deviation of $2 \mathrm{~mm}$ for the localization of landmarks.
\end{abstract}

Keywords: Deformable registration, tracking, coronary arteries, x-ray, computed tomography angiography, CTA

\section{Introduction}

Percutaneous Coronary Intervention (PCI) is a minimally procedure which is used to treat coronary artery narrowing. The workflow of the procedure is pretty standard. The physician intervenes on the patient under the guidance of an $\mathrm{x}$ ray imaging system. A guidewire is navigated in the diseased coronary artery. Before this interventional step, x-ray images with injection of a contrast agent are acquired to observe the shape of the vessels according to the projection angles which will be used for the intervention. During the guidewire navigation, the lesion is crossed and in some cases, the physician could benefit from a visual assessment of the coronary wall which may present plaques more or less calcified. The x-ray imaging interventional system used for per-operative guidance is not able to display this information mostly by lack of density resolution. On the contrary, Computed Tomography Angiography (CTA) is a modality which has the capability of capturing both the artery lumen and the characteristics of the vessel wall. To truly help physician to exploit this information during the course of the procedure, registering these two modalities would be useful.

$[3,1,9]$ have addressed this problem mostly in the case of images acquired at the same cardiac phase. In this situation and in a first analysis, the geometric 
difference between the two imaging situations can be described by a rigid transformation combined with a perspective projection since the CTA is a $3 \mathrm{D}$ dataset and the angiographic images correspond to a central projection with the x-ray source being the focal point. The following step is then to take into account the cardiac motion which may be captured or not in the CTA. If CTA can deliver multiple volumes corresponding at several phases of the cardiac cycle, it is at the expense of additional ionizing radiation to the patient. [2] has proposed to adapt a generic model of the cardiac motion to end-diastolic CTA dataset in order to register the two modalities along the cardiac cycle. This strategy raises the question of the validity of the generic model. In this work, we propose to explore another direction. The main interest of the physician is in the diseased vessel and he selects a projection angle by rotating the gantry such that the vessel of interest shows up relatively central in the image with limited foreshortening and minimum superimposition. Then to provide effective assistance during the guidewire navigation, the objective is to build a $3 \mathrm{D}$ deformation of the $3 \mathrm{D}$ vessel extracted from the CTA consistent with the apparent motion in the $2 \mathrm{D}$ angiographic sequence. So, any relevant information visible in the CTA volumes such as a plaque can be tracked in the $2 \mathrm{D}$ sequence. In [5], the proposed algorithm handles the non-rigid component of the cardiac motion by deformation in the image plane which is a simpification. In this work, we propose a method derived from [3] to track a coronary artery along the cardiac cycle with the objective of maintaining the consistency of the position along the vessels. We propose as in [6] to apply a 3D length preservation constraint as the coronary artery anatomically preserves its length along the cardiac cycle. In the following, we will describe the proposed method and explain the assessment strategy which includes metrics evaluating the registration and a specific metric related to the consistency of the position along the vessel.

\section{Method}

Before introducing the method, we first describe the data we have at hand. The 3D information is extracted from a Computed Tomography Angiography scan by a fully automated commercial product providing a segmentation of the coronary vessel structure. The coronary vessels are separated between the right and the left coronary and the different branches are represented by their centerlines which are represented by a tree $\mathcal{T}$ according to the anatomic structure of the vessels which separate in different branches at the bifurcations. From this structure we extract one vessel of interest $V$ which is tracked along the consecutive images of the x-ray record sequence. Even if the 3D model of the coronary vessels can be depicted by a tree, this may not be the case for the x-ray projection. Indeed, self superimpositions create crossings. The vessel segmentation may also cause over segmentation or miss some vessels. x-ray projections are segmented with an Hessian based vessel enhancement technique, and vessel like structures are extracted forming a set of curves which corresponds to the centerlines of the vessel [4]. The segmented object is organized in a graph by applying standard 
processing methods to connect neighboring centerlines. Considering the consecutive images obtained in the sequence of $N$ images by performing the acquisition after injection of the contrast agent, we obtain a set of graphs $\mathcal{G}=\left\{\mathcal{G}_{1}, \ldots, \mathcal{G}_{N}\right\}$.

We initiate the registration by identifying the initial rigid transformation, $T^{\circ}$ which maps $\mathcal{T}$ to the element $\mathcal{G}_{1} \in \mathcal{G}$ corresponding to the same diastolic cardiac phase as the pre-operative CT image [3].

The aim of the proposed tracking method is to track the vessel $V$ in all the consecutive phases of the cardiac motion, which necessitates to deform it. A spline description is a tool suited for this objective and the deformation can be represented by the optimization of its parameters, the control points. The registration itself is based on a two steps mechanism with first the determination of pairings between the projected curve describing the vessel $V$ and the centerlines represented through a graph structure. Second, the parameters are determined by minimizing an energy depending on the distance between the paired points and constraints on the vessel $V$.

\subsection{Problem modeling}

The 3D temporal tracking requires an a priori 3D model of vessels as introduced in $[11,7]$. They are represented by their centerline which is a $3 \mathrm{D}$ curve. The spline functions support a compact and smooth description of curves which can be continuously deformed by changing the position of the control points.

We thus fit an approximating cubic spline curve $C$ as in [8], using a centripetal method such that:

$$
\{C(u) \mid u \in[0,1]\} \approx V
$$

More precisely the spline is defined as:

$$
C(u)=\sum_{i=1}^{n} N_{i, p}(u) P_{i}
$$

where $N_{i, p}$ is the $i$ th B-spline of degree $p, P_{i}$ the $i$ th control point, $u$ the spline abscissa (between 0 and 1 ). Thanks to the choice of this model consecutive deformations can be represented by the optimization of the spline parameters. The set of control points to register the $3 \mathrm{D}$ vessel with the graph $\mathcal{G}_{t}$ is determined by solving this optimization problem:

$$
\hat{\mathcal{P}}_{t}=\operatorname{argmin}_{\mathcal{P} \in \mathbb{R}^{3 n}} E_{d}\left(C_{\mathcal{P}}, \mathcal{G}_{t}\right)+\beta E_{r}\left(C_{\mathcal{P}}\right)
$$

$t$ denotes the temporal index of the frame, $E_{d}()$ and $E_{r}()$ are respectively the data attachment and the regularization energy terms. In the following $\mathcal{P}_{t}$ denotes the set of control points for frame $t$ while $\mathcal{P}_{1}^{\text {init }}$ denotes the set of control points for the $3 \mathrm{D}$ vessel after the pose estimation $T^{\circ}$ for frame 1 . An initial position is used for the $3 \mathrm{D}$ vessel to build the data attachment term: it is the $3 \mathrm{D}$ vessel/spline $C_{\mathcal{P}_{1}^{\text {init }}}$ issued from the pose estimation for the first frame $t=1$ or $C_{\mathcal{P}_{t-1}}$ for frame $t>1$. For the sake of simplicity, $t$ will be omitted in the following. This $3 \mathrm{D}$ curve is projected onto the angiographic frame and is denoted $c$. A $2 \mathrm{D}$ curve $v$ corresponding to the projected $3 \mathrm{D}$ curve is extracted from the graph $\mathcal{G}$ as described in [3]. 
Data attachment term The data attachment term $E_{d}()$ is a sum of 3D residual distances issued from 3D to 2D pairings. The simplest method to build pairings is to use the closest neighbor scheme (as in the ICP). In [10], a variant of this approach is proposed: the idea is to represent the cardiac motion by covariance matrices on the different parameters describing the coronary tree. For this one, a generative 3D model is employed, i.e a model including a probabilistic distribution of position for the arterial segment. The concept of distance is then extended from standard Euclidean distance to Mahalanobis distance. This geometrically oriented analysis does not include the constraint of ordered pairing as proposed in [3] where it is shown that a point pairing that respects the order along paired curves yields better results than the closest neighbor scheme. Such an ordered pairing was obtained by the means of the Fréchet distance, that allows jumps between paired points. In presence of vessel deformation, we observed that the coherency of the obtained pairings can be discussed. So we propose to constrain the pairing construction with a $2 \mathrm{D}$ elongation preservation.

We first recall the Fréchet distance and its induced pairing [3]. Let $c=$ $\left\{c_{1}, \ldots, c_{n_{c}}\right\}$ and $v=\left\{v_{1}, \ldots, v_{n_{V}}\right\}$ be the $2 \mathrm{D}$ curves to be paired. The points $c_{i}$ are obtained as projection of points $C_{\mathcal{P}}\left(\bar{u}_{i}\right)$ from the $3 \mathrm{D}$ spline which represents the vessel. The points $v_{i}$ are the discrete points forming the centerline of the vessel extracted from the angiographic images. The point pairings are entirely defined by a single injective function $F: \mathbb{N} \rightarrow \mathbb{N}$. The Fréchet distance is defined as:

$$
\left\{\begin{array}{l}
F(1)=\operatorname{argmin}_{i_{v} \in I_{v}}\left\|v_{i_{v}}-c_{1}\right\| \text { with } I_{v}=\{1, \ldots, j u m p\} \\
F\left(i_{c}\right)=\operatorname{argmin}_{i_{v} \in I_{v}}\left\|v_{i_{v}}-c_{i_{C}}\right\| \text { with } I_{v}=\left\{F\left(i_{c}-1\right), \ldots, F\left(i_{c}-1\right)+j u m p\right\}
\end{array}\right.
$$

with jump a parameter controlling the length of allowed jumps in pairings. Looking at the pairing produced by this metric (as in Fig. 1, left), we observed that the simple application of the criteria of minimizing the pairing length may lead to irregular pairings. When computing rigid transformations as in [3], the least squares estimation introduces enough robustness to handle them. However, when dealing with non-linear transformations, the final result may be influenced. Inspired by the Fréchet distance, we present a pairing function which aims to build a pairing function that advances at the same speed along the $2 \mathrm{D}$ curves to be paired. Let consider a distance $d$ which will compute the length of $2 \mathrm{D}$ curves:

$$
d: \mathbb{N}^{2} \rightarrow \mathbb{R} \quad, \quad d\left(p_{1}, p_{2}\right)=\sum_{i=p_{1}+1}^{p_{2}}\left\|c_{i}-c_{i-1}\right\|
$$

We will define $F$ as:

$$
\left\{\begin{array}{c}
F(1)=\operatorname{argmin}_{i_{v} \in I_{v}}\left\|v_{i_{v}}-c_{1}\right\|^{2}+\lambda d\left(v_{1}, v_{i_{v}}\right)^{2} \text { with } I_{v}=\{1, \ldots, j u m p\} \\
F\left(i_{c}\right)=\underset{i_{c}}{\operatorname{argmin}} i_{i_{v} \in I_{v}}\left\|v_{i_{v}}-c_{i_{c}}\right\|^{2}+\lambda\left(d\left(v_{F\left(i_{c}-1\right)}, v_{i_{v}}\right)-d\left(c_{i_{c}-1}, c_{i_{c}}\right)\right)^{2} \\
\quad \text { with } I_{v}=\left\{F\left(i_{c}-1\right), \ldots, F\left(i_{c}-1\right)+j u m p\right\}
\end{array}\right.
$$

with $\lambda$ proportional to the local distance between the neighborhood of $i_{c}$ and $i_{v}$. This function favors point pairings between points which are approximately at the same distance from theirs respective neighborhoods. 
$F()$ provides $2 \mathrm{D}$ point pairings $\left(v_{F(i)}, c_{i}\right)$ between the 2D curves $v$ and $c$. To compute $3 \mathrm{D}$ deformations, we have to define $3 \mathrm{D}$ point pairings. $c_{i} \in c$ is associated to its corresponding $3 \mathrm{D}$ point $C_{\mathcal{P}}\left(\bar{u}_{i}\right)$. The $3 \mathrm{D}$ point $V_{F(i)}^{\prime}$ corresponding to $v_{F(i)}$ is the point from the backprojected line issued from $v_{F(i)}$ that is the closest to $C_{\mathcal{P}}\left(\bar{u}_{i}\right)$. The data attachment term is finally:

$$
E_{d}\left(C_{\mathcal{P}}, \mathcal{G}_{t}\right)=\sum_{i=1}^{n_{C}}\left\|V_{F(i)}^{\prime}-C_{\mathcal{P}}\left(\bar{u}_{i}\right)\right\|^{2}
$$
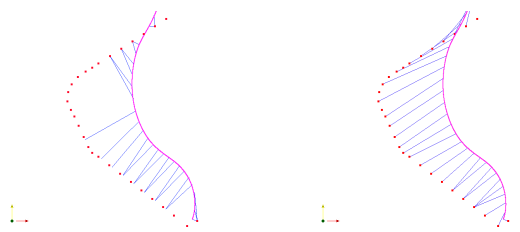

Fig. 1. The figure depicts pairings (blue) between projected $3 \mathrm{D}$ vessel $c$ (magenta) and $2 \mathrm{D}$ vessels $v$ (red), as observed locally on a case. On the left the parings are made with Fréchet, on the right with weighted Fréchet. Pairings are more regular with weighted Fréchet.

Regularization term The regularization term aims at minimizing the 3D elongation of $C$ :

$E_{r}\left(C_{\mathcal{P}}\right)=\sum_{j=1}^{J}\left(\left\|C_{\mathcal{P}}\left(e_{j}\right)-C_{\mathcal{P}}\left(e_{j-1}\right)\right\|-l_{j}\right)^{2}$ with $e_{j}=\frac{j}{J}$ and $l_{j}=\left\|C_{\mathcal{P}_{1}^{\text {init }}}\left(e_{j}\right)-C_{\mathcal{P}_{1}^{\text {init }}}\left(e_{j-1}\right)\right\|$

$J$ is the number of interval used to enforce the length constraint all along the vessel.

Energy minimization This global energy $E_{d}\left(C_{\mathcal{P}}, \mathcal{G}_{t}\right)+\beta E_{r}\left(C_{\mathcal{P}}\right)$ is minimized via a gradient descent. Thanks to the spline description of the $3 \mathrm{D}$ curve, the analytic expression of the gradient is used for the gradient descent. The pairings are recomputed along the descent every 1000 iterations. The minimization is stopped when the gradient norm is below a threshold, whose value has been chosen in preliminary experiments.

\section{Performance evaluations}

Qualitative evaluation of the performance of the proposed algorithms can first be done by a visual control of the deformation of the projected deformed vessel 
over the angiographic image along the cardiac cycle. We also propose three quantitative measures. The first two corresponds to methodological expectations on the performance but does not cover directly the intended clinical application. The third one replicates more closely the expectations from a clinical standpoint.

\section{$3.12 \mathrm{D}$ curve distance}

This is an indirect measure of the quality originally proposed in [11]. Its intent is not to evaluate the correctness of the selected vessel in the angiographic image. This measure indicates only if the deformation of the vessel $V$ has adapted well to the observed projection in the angiographic image. For each registration the distance between the projected curve $\mathcal{P}(C)$ and its corresponding $2 \mathrm{D}$ vessel $v$ is computed, with $\mathcal{P}$ the projection matrix. Our measure of $2 \mathrm{D}$ curve distance is:

$$
c d=\frac{1}{n_{C}} \sum_{i=1}^{n_{C}}\left\|\mathcal{P}(C(i))-v_{\text {closest }(i)}\right\|
$$

with closest $(i)$ the index in $v$ of to the closest point to $\mathcal{P}(C(i)), n_{C}$ the number of retained sample points in $C$. $C$ is a continuous curve defined by an analytic representation based on spline. For this evaluation measure and also the next one, we select a number of points along the curve. We take them equally spaced.

\subsection{Shape preservation}

For this analysis, we start from the idea that the vessel shall return to its initial state if the tracking is performed on a series of consecutive images which start and end by the same image. Let $N$ the number of angiographic images in a sequence which covers a cardiac cycle, the tracking is done from the frame 1 to the frame $N$, resulting in $N$ 3D curves corresponding to the same vessel of interest temporally tracked, $C=\left\{C_{1}, \ldots, C_{N}\right\}$. One can then generate the reverse sequence starting from image $N-1$ down to image 1 and continue to apply the tracking algorithm. The result is an other set of $3 \mathrm{D}$ curves $C^{\prime}=$ $\left\{C_{N-1}^{\prime}, \ldots, C_{1}^{\prime}\right\}$. The similarity of the curves $C_{k}^{\prime}$ and $C_{k}$ is an indirect measure of the performance of the tracking algorithm. To measure the similarity, we chose to compute the distance between $C_{1}$ and $C_{1}^{\prime}$ which are respectively the first and last curves of the forward and backward tracking. Our measure of shape preservation is:

$$
s p=\frac{1}{n_{C}} \sum_{i=1}^{n_{C}}\left\|C_{1}(i)-C_{1}^{\prime}(i)\right\|
$$

with $n_{C}$ the number of points in $C$ and $C^{\prime}$.

\subsection{Landmark tracking}

From the point of view of the clinical application, this is the most important measure. The idea is to evaluate if a location defined along the coronary vessel 
is correctly tracked with the beating heart. A location in the vessel $V$ is defined by its curvilinear abscissa. In the angiographic image, identifying a fixed point is more challenging.

To do so, we first manually point an easily identifiable landmark along the $2 \mathrm{D}$ vessel that correspond to the $3 \mathrm{D}$ vessel of interest. Vessel bifurcations are natural candidates for such landmarks, and we manually have pointed one bifurcation along the x-ray sequence for each 3D vessel to be registered. To decide whether the same 3D point of the tracked vessel is paired to this ground truth, we use the curvilinear abscissas $u$ (along the spline) of the paired 3D points to the bifurcation. A perfect tracking (along with a perfect manual identification of the bifurcation) should yield the same curvilinear abscissa for all paired 3D points, thus the standard deviation of all curvilinear abscissas is an adequate measure to assess the tracking.

Formally, let $U=\left\{u_{1}, \ldots, u_{N}\right\}$ be the $N$ abscissas along the x-ray sequence of the paired 3D points, eg. $C\left(u_{t}\right)$ is paired with the bifurcation/landmark in frame $t$, and $\bar{u}$ be the average value over $U$, the proposed measure is

$$
l t=\sqrt{\frac{1}{N} \sum_{i=1}^{N}\left(u_{i}-\bar{u}\right)^{2}}
$$

\section{Results}

To assess the performance of the proposed approach, we use anonymous data collected after informed patient consent for use in this type of investigation. These data come from five different patients. Both the CT scan and the angiographic images are available. We selected in the angiographic sequences a sub-sequence of 20 images which covers a full cardiac cycle or a bit more depending on the patient case. The $\mathrm{CT}$ scans have been pre-processed to extract the coronary vessel trees as described above. Several 3D vessels may have been selected for a given patient, yielding a total of 10 different tracking experiments. Each of them is analyzed separately from the other. Selection is based on the available angiographic views and the vessels are selected as the ones that could be the object of an interventional procedure. In the following, we propose to compare three different settings of the proposed algorithms: the standard Fréchet approach to determine pairings between two curves, the weighted Fréchet as described above with and without the constraint on the length of the vessel $V . \lambda$ has been set to $\frac{l_{d}^{2}}{d_{p}(c)^{2}}$, with $l_{d}$ the local distance between neighborhoods of two points and $d_{p}(c)$ the average distance between points in $c$ curve. To tune $\beta$ we observed the length variations of the deforming vessel (see Fig. 2) and the data attachment term value at convergence for different values of $\beta$ on few cases. First, we observe for $\beta \geq 100$ a variation smaller than $0.1 \%$ despite an apparent $2 \mathrm{D}$ length variation on the fluoroscopic plane of $5 \%$. Second, we notice that the data attachment term value at convergence remain the same for every tested values of $\beta$, we thus conclude that a big $\beta$ does not alter the closeness of the two curves. Considering 
this last point and the fact that the length constraint corresponds to the exact physical behavior of the coronary during the contraction, we set $\beta=1000$ such that the deformed vessel keeps a constant length.

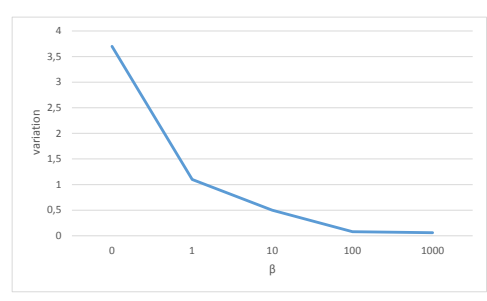

Fig. 2. Variation of the 3D length of a vessel depending on the $\beta$ parameter, expressed as a percentage of the initial $3 \mathrm{D}$ length.

Fig. 3 is an example of the obtained results in one case. The average execution time per image is 30 seconds on an Intel R Core TM i7-4712HQ CPU on a virtual machine. The code has not been optimized.

\subsection{D curve distance}

As explained in section 3.3, we have computed the $2 \mathrm{D}$ curve distance for the different considered cases. The obtained results are displayed in Fig. 5 with the weighted Fréchet approach. The values fall in the range of $0.15 \mathrm{~mm}$ with a maximum of $0.25 \mathrm{~mm}$. In this figure, we display for each frame from 1 to 20 the value of the distance (Eq. 6). Contrary to [11], we do not observe a variation with the cardiac cycle. So we draw the conclusion that the deformation capability of our 3D vessel is sufficient to follow the deformation of the arteries. In all the considered cases through observations by an experienced reader, we have validated that the tracked arteries are the correct ones. The application of the length constraint does not restrain the capability of the model to deform.

\subsection{Shape preservation}

The objective of this measure is to evaluate the correctness of the deformation of this vessel by looking at a case for which the ground truth is known by design of the test. Left of Tab. 1 presents the results obtained on our ten different cases. When the length constraint is not applied, the results show that the deformation is not well controlled. In the first case, we are close to about $40 \mathrm{~mm}$ of difference between the two curves. This is to be compared with the typical length of a coronary vessel that is about $80 \mathrm{~mm}$. The two other techniques which apply the 3D length constraint give similar results. Overall the average 3D distance 

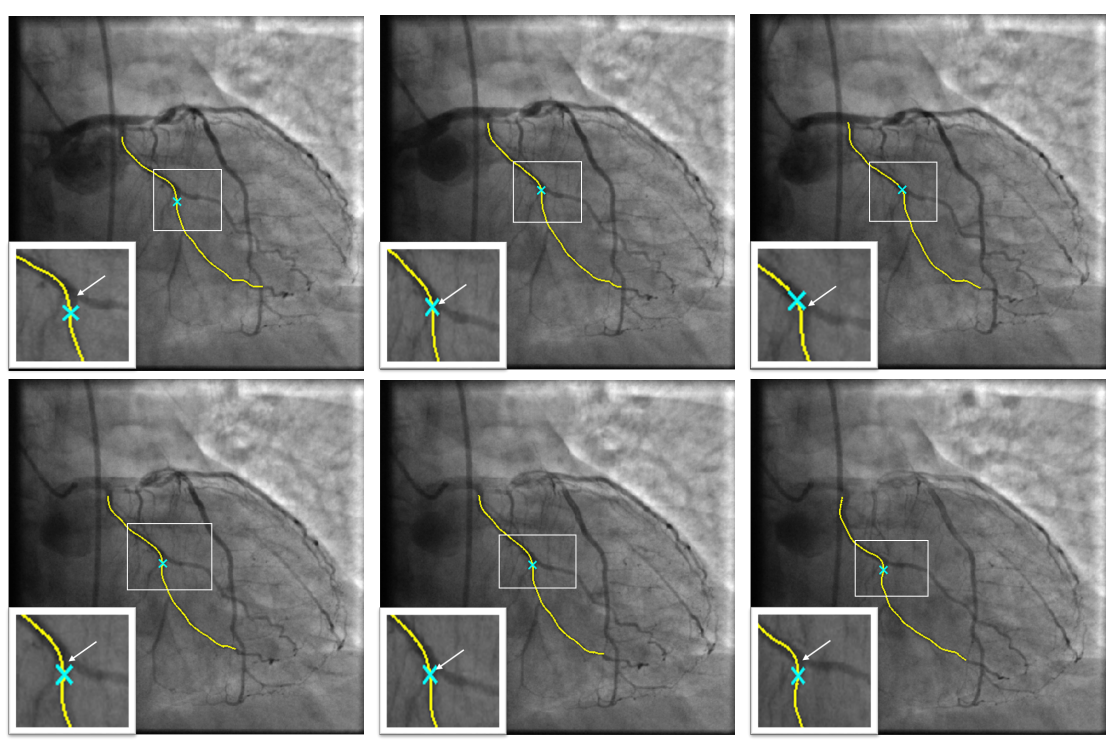

Fig. 3. Tracking results for one patient over one cardiac cycle. The yellow curve represents the projected 3D vessel, the blue cross represents the point tracked as the bifurcation, and the white arrow designs the bifurcation as marked by an experienced reader. Those images come from a 20 frames sequence. This figure shows the frames 1 , $5,9,13,17,20$, from left to right, up and down.
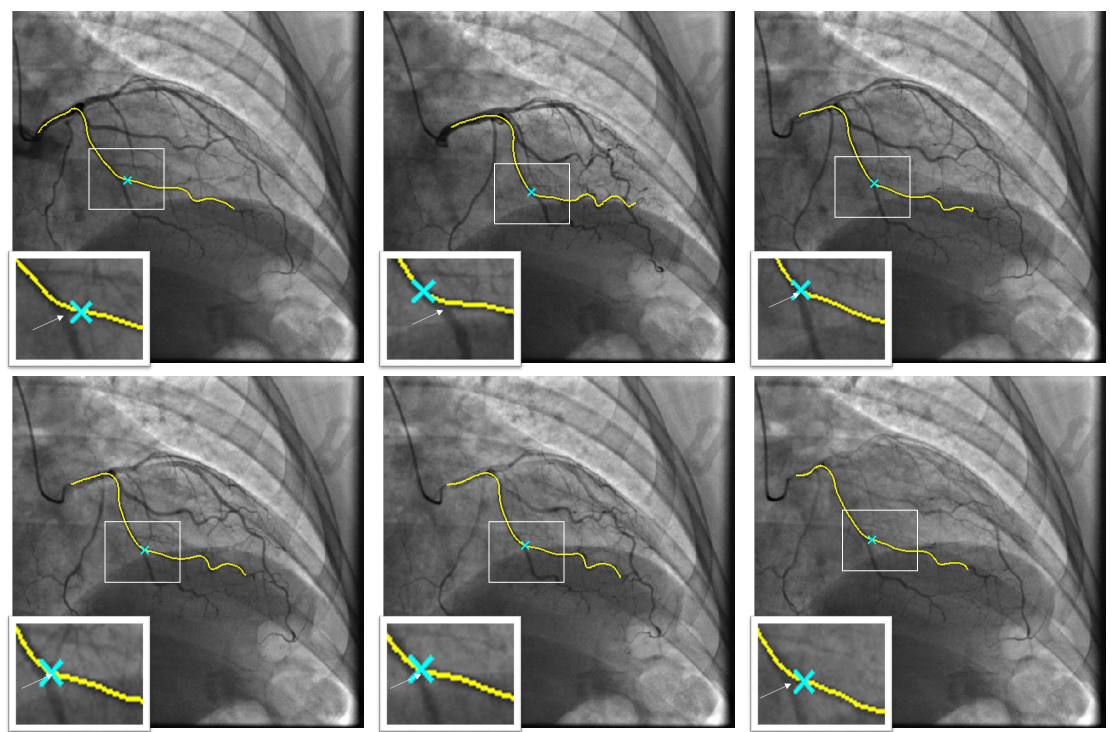

Fig. 4. Tracking results for a second patient. Same conventions than in Fig. 3 


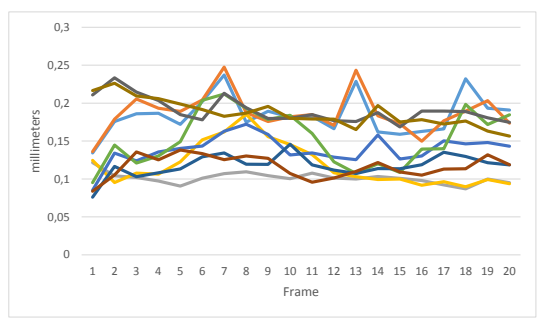

Fig. 5. The $2 \mathrm{D}$ curve distance measure ( $c$, see Eq. 6), for the ten cases. The values are plotted for the 20 successive frames of the selected sub-sequence.

between the two curves after a tracking over 40 projections is inferior to $7 \mathrm{~mm}$ which is pretty encouraging. We observe some variations from case to case, which are very likely to be caused by the complexity of the motion. For some patients, the motion is mostly a translation and a rotation in the image plane plus some large scale contraction. In some other cases, the arteries are more tortuous and along the cardiac cycle they can fold/unfold. These cases are more challenging.

\begin{tabular}{c|c|c|c||cc|c}
\hline \multicolumn{3}{c||}{ Shape preservation } & \multicolumn{3}{c}{ Landmark tracking } \\
\hline Vessels & Fréchet & $\begin{array}{c}\text { Weighted } \\
\text { Fréchet }\end{array}$ & $\begin{array}{c}\text { Weighted Fréchet } \\
\text { WLC* }\end{array}$ & Frchet & $\begin{array}{c}\text { Weighted } \\
\text { Fréchet }\end{array}$ & $\begin{array}{c}\text { Weighted Fréchet } \\
\text { WLC* }\end{array}$ \\
\hline 1 & 7.96 & 7 & 39.81 & 1.73 & 2.21 & 14.5 \\
2 & 11.11 & 8.7 & $\mathrm{x}$ & 2.64 & 3.2 & $\mathrm{x}$ \\
3 & 2.19 & 1.4 & 1.43 & 1.72 & 2.1 & 1.01 \\
4 & 4.21 & 3 & 2.44 & 2.62 & 1.3 & 1.14 \\
5 & 1.8 & 1.8 & 5.02 & 1.12 & 0.7 & 2.75 \\
6 & 7 & 3.18 & 4.9 & 3.88 & 4.1 & 2.79 \\
7 & 4.94 & 7.3 & 6.4 & 1.9 & 2.4 & 1.38 \\
8 & 2.62 & 1.7 & 1.92 & 1 & 1.2 & 1.12 \\
9 & 11.5 & 9.6 & 8.43 & 2.1 & 2.1 & 2.52 \\
10 & 10.1 & 10.5 & 7.92 & 1.63 & 1.9 & 3.01 \\
average & 6.33 & 5.42 & 8.68 & 2.02 & 2.11 & 3.36 \\
\hline
\end{tabular}

Table 1. Results in millimeters of the 3 methods for shape preservation and landmark tracking evaluations on 10 vessels from 5 patients. ${ }^{*} \mathrm{WLC}=$ Without Length Constraint

\subsection{Landmark tracking}

The results obtained with this method are presented on the right of Tab. 1. The presented value is the standard deviation of the set of curvilinear abscissa for 
the point associated to the bifurcation manually marked in each projection. The average value is 2.02 and 2.11 for the two methods with the 3D length constraint. When the length constraint is not applied, we have a larger value as anticipated. This observed standard deviation accounts for two sources of errors: the error created by the algorithm and the error on the ground truth. Marking the exact location of a bifurcation in x-ray image is difficult since the bifurcated vessel may superimpose to the main branch and cannot be distinguished from it. The length of this superimposition varies along the cardiac cycle. This problem is also more or less pronounced depending on the apparent angle at the level of the bifurcation.

\section{Discussion and conclusion}

We have presented a method to track a coronary artery from 3D to 2D. Starting from a first registration of the complete tree obtained at the same phase, we then focus our attention on a single vessel in the idea of mimicking the interventional procedure whose objective is to treat a diseased vessel. The key point of the algorithm approach that we have proposed is to establish pairings using the Fréchet algorithm. These pairings are done between the projected 3D vessel and the centerlines that were segmented in the angiographic image. The $3 \mathrm{D}$ vessel is then deformed to minimize the total pairing length in the 3D space and under the constraint of length preservation. This constraint is meaningful because this property is respected by the coronary vessel along the cardiac cycle and because it is applied to the 3D curve and not its projection. In the evaluation, we have observed that the absence of this constraint degrades the result even if the tracking remains apparently correct. The individual trajectories of points are not constrained a priori to be smooth. In the evaluation, we have looked a posteriori at the trajectory of specific points placed at the bifurcations and we found that their projections follow pretty well the apparent movement of the bifurcations in the x-ray projections. We have also evaluated the Fréchet pairing algorithm and a variant. Main difference is the introduction of an additional contributor in the optimized criteria. The criteria is based on the variation of the apparent length in the successive pairings. As observed in Fig. 1, the obtained pairings are more meaningful than with the standard Fréchet method. Looking at the results on the ten test cases and with the different evaluation strategies implemented, this difference translate in an improvement for the shape preservation criteria. Interestingly the average distance is about $5.5 \mathrm{~mm}$ after a tracking performed over 40 frames. Typical length of the selected coronary arteries is about $80 \mathrm{~mm}$. For the landmark tracking, the average value is about $2 \mathrm{~mm}$ in both variants of the Fréchet method which also accounts for the imprecision in the definition of the bifurcation location in the angiographic image. This last result appears very encouraging since it is in the range of the expectation of the physician who expects to get information on the vessel wall at the place where the tip of the guidewire is positioned. The exact position in the artery of the guidewire tip is also changing slightly with the cardiac motion. In a further step, methods to 
register the position of the guidewire tip observed in the subsequent fluoroscopic sequence with the angiographic projection will be developed. A more complete evaluation of the accuracy could also be done by performing in parallel to the angiographic acquisition some intravascular images which are able to display the vessel wall properties. After proper co-registration of these intravascular images with the angiographic images, it would be then possible to compare the two modalities intravascular and $\mathrm{CT}$ views of the vessel wall and to quantify the geometrical differences from an algorithmic standpoint.

\section{References}

1. Timur Aksoy, Gozde Unal, Stefanie Demirci, Nassir Navab, and Muzaffer Degertekin. Template-based CTA to x-ray angio rigid registration of coronary arteries in frequency domain with automatic x-ray segmentation. Medical physics, 40(10), 2013.

2. Nora Baka, CT Metz, Carl Schultz, Lisan Neefjes, Robert Jan van Geuns, Boudewijn PF Lelieveldt, Wiro J Niessen, Theo van Walsum, and Marleen de Bruijne. Statistical coronary motion models for $2 \mathrm{D}+\mathrm{t} / 3 \mathrm{D}$ registration of $\mathrm{x}$-ray coronary angiography and CTA. Medical image analysis, 17(6):698-709, 2013.

3. Thomas Benseghir, Grégoire Malandain, and Régis Vaillant. A tree-topology preserving pairing for 3D/2D registration. International Journal of Computer Assisted Radiology and Surgery, 10(6):913-923, 2015.

4. Alejandro F Frangi, Wiro J Niessen, Koen L Vincken, and Max A Viergever. Multiscale vessel enhancement filtering. In International Conference on Medical Image Computing and Computer-Assisted Intervention, pages 130-137. Springer, 1998.

5. Carlo Gatta, Simone Balocco, Victoria Martin-Yuste, Ruben Leta, and Petia Radeva. Non-rigid multi-modal registration of coronary arteries using siftflow. In Iberian Conference on Pattern Recognition and Image Analysis, pages 159-166. Springer, 2011.

6. Martin Groher, Darko Zikic, and Nassir Navab. Deformable 2D-3D registration of vascular structures in a one view scenario. IEEE Transactions on Medical Imaging, 28(6):847-860, 2009.

7. Tim Hauke Heibel, Ben Glocker, Martin Groher, Nikos Paragios, Nikos Komodakis, and Nassir Navab. Discrete tracking of parametrized curves. In IEEE Conference on Computer Vision and Pattern Recognition. CVPR., pages 1754-1761. IEEE, 2009 .

8. Les Piegl and Wayne Tiller. The NURBS book. Springer Science \& Business Media, 2012.

9. Daniel Ruijters, Bart M ter Haar Romeny, and Paul Suetens. Vesselness-based 2D$3 \mathrm{D}$ registration of the coronary arteries. International journal of computer assisted radiology and surgery, 4(4):391-397, 2009.

10. Eduard Serradell, Adriana Romero, Rubén Leta, Carlo Gatta, and Francesc Moreno-Noguer. Simultaneous correspondence and non-rigid 3d reconstruction of the coronary tree from single x-ray images. In IEEE International Conference on Computer Vision (ICCV), 2011, pages 850-857. IEEE, 2011.

11. Guy Shechter, Frédéric Devernay, Eve Coste-Maniere, and Elliot R McVeigh. Temporal tracking of 3D coronary arteries in projection angiograms. In Medical Imaging 2002, pages 612-623. International Society for Optics and Photonics, 2002. 